\title{
Comparison of two in-laboratory titration methods to determine effective pressure levels in patients with obstructive sleep apnoea
}

\author{
M P Bureau, F Sériès
}

\begin{abstract}
Background-Treatment of the sleep apnoea/hypopnoea syndrome with nasal continuous positive airway pressure (CPAP) conventionally requires a titration procedure in a sleep laboratory. The upper airway has a hysteresis phenomenon which accounts for a decrease in the effective positive pressure level once an initial effective pressure setting has been reached. The aim of this study was to quantify the difference in the initial and final effective pressure settings when the titration sleep study takes into account these possible changes in the effective pressure level.
\end{abstract}

Methods-Eighty five patients completed the study. During a titration sleep study the pressure was increased by $1 \mathrm{~cm} \mathrm{H}_{2} \mathrm{O}$ in a stepwise fashion until obstructive respiratory events disappeared (effective pressure 1, Peff $)$. The pressure level was then decreased by increments of $1 \mathrm{~cm} \mathrm{H}_{2} \mathrm{O}$ until breathing abnormalities reappeared. At this time pressure was re-increased by increments of $1 \mathrm{~cm} \mathrm{H} \mathrm{H}_{2} \mathrm{O}$ to normalise breathing $\left(\mathbf{P e f f}_{2}\right)$.

Results-The mean (SD) value of Peff was $9.5(2.6) \mathrm{cm} \mathrm{H}_{2} \mathrm{O}$. The pressure was then reduced during $0.5(0.6)$ hours to reach the minimal pressure $\mathbf{( 7 . 0}$ $\left.(2.0) \mathrm{cm} \mathrm{H}_{2} \mathrm{O}\right)$. The pressure obtained after a downward titration had to be re-increased in 79 patients, the Peff, level being significantly lower than Peff $_{1}$ (8.9 (2.8) $\left.\mathrm{cm} \mathrm{H}_{2} \mathrm{O}, \mathrm{p}=0.0002\right)$, mean difference $0.6(1.5) \mathrm{cm} \mathrm{H}_{2} \mathrm{O}(95 \%$ confidence interval 0.29 to 0.93 ).

Conclusion-Attempts to decrease the positive pressure level during conventional determination of the effective pressure level allow a significant decrease in the pressure setting. This should be taken into account in each patient who requires an in-laboratory manual CPAP titration procedure.

(Thorax 2000;55:741-745)

Keywords: sleep apnoea/hypopnoea syndrome; continuous positive airway pressure (CPAP); pressure settings

Correspondence to: Dr F Sériès

email:

frederic.series@med.ulaval.ca

Received 5 January 2000

Returned to authors

9 March 2000

Revised version received

17 April 2000

Accepted for publication

5 June 2000 important to initiate treatment once a diagnosis has been made. Different therapeutic approaches have been used. Patients are first encouraged to lose weight. ${ }^{6}$ Surgical procedures such as uvulopalatopharyngoplasty can be performed but are not very effective with a success rate of about $30 \%{ }^{7}$ Anterior mandibular positioners can also be tried in patients with mild to moderate disease, ${ }^{8}$ but nasal continuous positive airway pressure (CPAP) represents the most effective treatment of SAHS. The usefulness of this treatment was shown by Sullivan et al in 1981. ${ }^{9}$ Various side effects such as machine and mask leakage, irritation of the eyes resulting from air leaks, nasal and pharyngeal dryness, and rhinorrhoea have all been reported during CPAP treatment and can lead to poor treatment compliance and discontinuation of treatment. ${ }^{1011}$ This is undesirable because, if the patient interrupts the use of CPAP, the neuropsychological consequences of SAHS immediately recur. ${ }^{12}$ It is therefore particularly important to determine the minimal pressure to be set for home CPAP treatment in order to increase comfort and treatment acceptability.

The effective positive pressure level is the one that abolishes obstructive apnoea, hypopnoea, snoring, and respiratory related arousals in all body positions and sleep stages. This pressure level can be determined by different methods. The conventional approach is to ask the attending technician to adapt the positive pressure level manually to reach these goals during a complete night in the sleep laboratory. An alternative to the full night titration method is a split night sleep study during which the first half of the night is spent without CPAP to confirm the diagnosis and the second half is performed with CPAP to find the adequate pressure level. ${ }^{13}{ }^{14}$ More recently, autotitrating CPAP machines have been developed that allow continuous automatic adjustment of the pressure level depending on patient needs in various body positions and sleep stages. ${ }^{15}$ These machines are currently used in hospital facilities but do not require technician attendance.

It has recently been shown that the upper airway flow regimen is subject to a hysteresis phenomenon during CPAP treatment. ${ }^{16}$ During upward titration the positive pressure level at which flow limitation disappears is 2-5 $\mathrm{cm} \mathrm{H}_{2} \mathrm{O}$ higher than the level at which it reappears during downward titration. These upper airway properties have recently been used to determine more precisely the effective 
positive pressure level in SAHS, ${ }^{17-19}$ a downward pressure adjustment being achieved once the initial conventional optimal pressure level has been reached. The difference in the effective pressure levels obtained with or without taking into account the upper airway mechanical properties has not been measured. We hypothesised that the effective pressure level measured with this secondary downward titration procedure should be significantly lower than with the conventional method. The aim of this study was therefore to compare the effective positive pressure obtained by these two titration methods.

\section{Methods}

PATIENTS

Data for the study were collected between June 1998 and June 1999. Eighty five consecutive patients (74 men) aged 29-74 years were included. At the time of the study patients were either already being treated with CPAP at home $(n=12)$ or were experiencing CPAP titration for the first time $(\mathrm{n}=73)$. The 12 patients already on CPAP required a second titration procedure because of a clinical suspicion of an inadequate pressure setting. In all cases the clinical diagnosis had been confirmed by the results of a sleep recording study (home oximetry or conventional polysomnography). The oximetric tracing was considered to be characteristic of SAHS when the desaturation index was $>15 /$ recording hour. ${ }^{20}{ }^{21}$ If this criterion was not met in a patient clinically suspected of having SAHS, a polysomnographic study was performed to confirm the diagnosis.

\section{CPAP TITRATION}

CPAP titration was performed during continuous electroencephalographic (EEG) monitoring $\left(\mathrm{C}_{4} / \mathrm{A}_{1}, \mathrm{C}_{3} / \mathrm{A}_{2}, \mathrm{O}_{2} / \mathrm{A}_{1}, \mathrm{O}_{1} / \mathrm{A}_{2}\right)$, submental and anterior tibialis electromyography, electrocardiography, electro-oculography, nasal flow with a pneumotachograph, mouth flow with a thermistor, thoracoabdominal movements with inductance plethysmography, breathing sounds with two microphones placed at the head of the bed, oxyhaemoglobin saturation with an ear oximeter and mask pressure. All variables were recorded on a computer (Sandman, Melville Diagnostics, Ottawa, Ontario, Canada).

The initial CPAP setting was $4 \mathrm{~cm} \mathrm{H}_{2} \mathrm{O}$. The technician then increased the pressure progressively by increments of $1 \mathrm{~cm} \mathrm{H}_{2} \mathrm{O}$ until obstructive apnoea, hypopnoea, snoring, and flow limited breathing associated with arousals disappeared. Obstructive events were identified by the presence of increased thoracoabdominal movements and/or paradoxes associated with

Table 1 Anthropometric characteristics of the study population

\begin{tabular}{lccc}
\hline & $\begin{array}{l}\text { Total subjects } \\
(n=85)\end{array}$ & $\begin{array}{l}\text { Subjects diagnosed } \\
\text { by oximetry }(n=43)\end{array}$ & $\begin{array}{l}\text { Subjects diagnosed } \\
\text { by polysomnography } \\
(n=42)\end{array}$ \\
\hline Age & $50.0(10.7)$ & $50.5(10.5)$ & $49.5(10.9)$ \\
Weight $(\mathrm{kg})$ & $107.6(24.8)$ & $111.8(27.3)$ & $103.3(21.4)$ \\
Height $(\mathrm{m})$ & $1.7(0.1)$ & $1.7(0.1)$ & $1.7(0.1)$ \\
Body mass index $\left(\mathrm{kg} / \mathrm{m}^{2}\right)$ & $37.3(8.4)$ & $38.8(8.8)$ & $35.9(7.8)$ \\
Neck circumference $(\mathrm{cm})$ & $43.7(3.8)$ & $44.4(3.6)$ & $43.0\left(7.8_{-}\right.$ \\
\hline
\end{tabular}

abolition or decrease in instantaneous flow. Arousals were defined according to standard criteria. $^{22}$ This pressure level was identified as effective pressure 1 ( Peff $\left._{1}\right)$. The pressure was then reduced by $1 \mathrm{~cm} \mathrm{H}_{2} \mathrm{O}$ increments every 2-5 minutes until one of the above mentioned breathing abnormalities reappeared or the minimal pressure level $\left(4 \mathrm{~cm} \mathrm{H}_{2} \mathrm{O}\right)$ was reached. The minimal pressure reached before the pressure re-increased was identified as Pmin. The technician then increased the pressure again by $1 \mathrm{~cm} \mathrm{H}_{2} \mathrm{O}$ every time a new obstructive event appeared. The second pressure level that resulted in the abolition of abnormal respiratory events and snoring was identified as effective pressure $2\left(\mathrm{Peff}_{2}\right)$ and was applied for the rest of the night. The downward titration procedure was done only when Peff $_{1}$ was $\geqslant 6 \mathrm{~cm} \mathrm{H}_{2} \mathrm{O}$ and when the recording time required to reach $\mathrm{Peff}_{1}$ was less than five hours.

Since the effective pressure should abolish breathing abnormalities in every sleep stage and sleep position, as far as possible, each titration procedure was obtained with the subjects reaching REM sleep and sleeping supine. In the morning the whole recording was reviewed by the respiratory physician in charge of the patient to verify the pressure setting. This was then prescribed for chronic CPAP treatment at home.

DATA AND STATISTICAL ANALYSIS

The latency for Peff 1 corresponds to the time between the onset of sleep and Peff . Peff $_{2}$ latency was measured as the time necessary to reach Peff $_{2}$ from Pmin. Numeric variables such as anthropometric and polysomnography data were summarised using means and standard deviations. An unpaired $t$ test was used to compare Peff values between patients diagnosed by oximetry and polysomnography. A randomised block design was used to compare the minimal and effective pressure values. The relationship between Peff $_{2}$ or Pmin and Peff 1 was examined by least squares regression analysis. $p$ values of $<0.05$ were considered significant.

\section{Results}

The characteristics of the participating subjects are shown in table 1 . Body mass index (BMI) and neck circumference were slightly greater in patients diagnosed by oximetry than in those diagnosed by polysomnography $(p=0.09$ and 0.1 , respectively). Peff 1 was consistently higher in the former group (10.0 (2.5) $\mathrm{cm} \mathrm{H}_{2} \mathrm{O}$ and 8.9 (2.5) $\mathrm{cm} \mathrm{H}_{2} \mathrm{O}$, respectively, $\left.\mathrm{p}=0.05\right)$. Table 2 summarises the results of the polysomnographic studies. Effective pressure levels did not differ between men and women.

Table 2 Sleep and breathing characteristics of patients diagnosed by polysomnography $(n=42)$

\begin{tabular}{ll}
\hline Total sleep time (h) & $5.5(1.7)$ \\
Stage I-II (\% total sleep time) & $74.3(15.0)$ \\
Stage III-IV (\% total sleep time) & $13.3(9.2)$ \\
REM sleep (\% total sleep time) & $13.0(8.2)$ \\
Arousal index (no/h) & $40.5(22.8)$ \\
Apnoea + hypopnoea index (no/h) & $40.5(27.6)$ \\
Apnoea + hypopnoea index supine (no/h) & $54.5(31.2)$ \\
\hline
\end{tabular}

Values are mean (SD) 


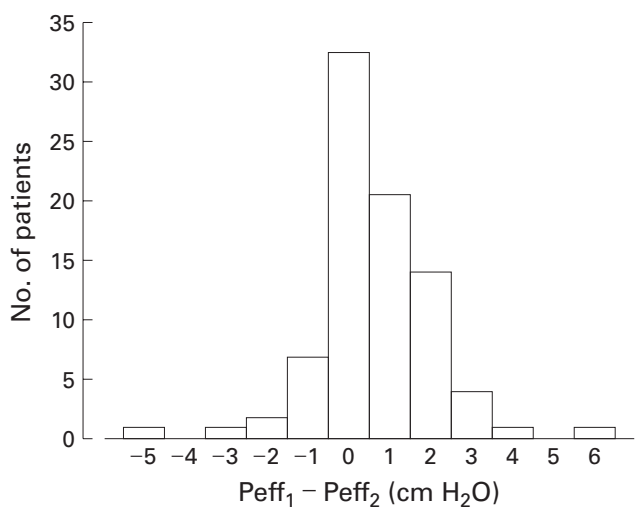

Figure 1 Repartition of patients with different values of Peff - Peff.

The value of $\mathrm{Peff}_{1}$ was 9.5 (2.6) $\mathrm{cm} \mathrm{H}_{2} \mathrm{O}$ with a latency for Peff ${ }_{1}$ determination of 2.4 (1.3) hours. This pressure was maintained for 1.3 (1.0) hours until the optimal conditions for determination of effective pressure (supine in REM sleep) had been reached. These two conditions were fulfilled in 79 patients. In all patients the positive pressure level could be decreased following determination of Peff $_{1}$. The Pmin value averaged $7.0(2.0) \mathrm{cm} \mathrm{H}_{2} \mathrm{O}$ and was reached in $0.5(0.6)$ hours. A reincrease in the positive pressure level was needed in 79 patients (these were not necessarily the same patients as those in the previously mentioned group of 79 patients). This reincrease was caused by snoring $(n=26)$, flow limitation associated with arousals $(n=32)$, obstructive hypopnoea $(\mathrm{n}=19)$, and obstructive apnoea $(\mathrm{n}=2)$ with a $\mathrm{Peff}_{2}$ value of 8.9 (2.8) $\mathrm{cm} \mathrm{H}_{2} \mathrm{O}$. Peff 2 was significantly lower than $\operatorname{Peff}_{1}(\mathrm{p}<0.0001)$ with a mean difference of 0.6 (1.5) $\mathrm{cm} \mathrm{H}_{2} \mathrm{O}$ (95\% confidence interval 0.29 to 0.93 ). The decrease in pressure was achieved in $48 \%$ of patients (mean (SD) $\mathrm{Peff}_{2}-\mathrm{Peff}_{1} \quad 1.7 \quad$ (1.0) $\mathrm{cm} \mathrm{H}_{2} \mathrm{O}$ (range 1-6 $\mathrm{cm} \mathrm{H}_{2} \mathrm{O}$ ), fig 1). Interestingly, the difference in Peff was significant only in patients who were not already on CPAP and was not significant in those currently being treated with CPAP at home.

This pressure was applied for the rest of the night (2.5 (1.5) hours). The latency of Peff was 1.2 (1.2) hours which was significantly shorter than the latency of Peff $1(p<0.0001)$. A positive correlation was found between the difference in Peff ${ }_{1}$ and Pmin and the Peff, value (p $=0.0001, R=0.42$ ). However, there was a closer correlation between Peff 1 and Peff ${ }_{2}$ with a correlation coefficient of $0.85(p=0.0001)$. In six patients no increase in pressure was required once Pmin was reached as no obstructive breathing abnormalities were observed. All six patients had REM sleep in the supine position at Pmin. The apnoea hypopnoea index (AHI) was 4.8 (5.6)/hour with an arousal index of $15.6(9.4) /$ hour for the whole CPAP sleep study.

\section{Discussion}

This prospective study shows that the effective pressure level can be significantly decreased using a downward titration procedure during conventional CPAP titration. The study population is representative of the patients with SAHS referred to our sleep laboratory and who undergo a CPAP titration procedure. Since our diagnosis and the CPAP titration procedure used are in accordance with recommended criteria, ${ }^{23}$ we believe that these results are valid for the general SAHS population.

It could be argued that changes in sleep state architecture and in body position between the initial and final pressure rise could result in a difference in upper airway collapsibility and therefore in Peff. However, only three patients did not have REM sleep or slept supine during determination of Peff ${ }_{2}$. Furthermore, it should be emphasised that $\mathrm{Peff}_{2}$ was applied for 2.5 hours, providing a sufficiently long recording time to allow recurrent breathing disturbances to eventually reappear. From a technical point of view, human error due to lack of attention by the technician or to inadequate identification of breathing abnormalities cannot account for our findings since the adequacy of the pressure setting was checked by the physician in charge of the study. For these reasons we believe that the observed difference in Peff is not the result of methodological biases.

According to our all night results, the positive pressure level applied abolished sleep related breathing disorders during the titration night. This is in accordance with the results of previous studies using a similar titration protocol. ${ }^{17-19}$ It could be asked to what extent subsequent nights would be normal with the CPAP level set at Peff ${ }_{2}$ when not preceded by a higher pressure level. However, it is clear from previous studies that no residual abnormal breathing events are observed on control CPAP sleep studies when a titration protocol similar to ours is used. With the Autoset machine the recommended pressure setting is the 90th percentile of the pressure history. ${ }^{19}$ Respiratory variables measured at this pressure setting are normal after three and eight months of CPAP treatment. ${ }^{24}$ Similarly, using the Autoadjust auto CPAP device, Stradling et al found that setting the CPAP machine at the level that abolished most of the obstructive events, but that was not necessarily the highest pressure ever reached, provided a similar clinical improvement to conventional CPAP titration. ${ }^{25}$ We are therefore confident that a second sleep recording performed at a constant Peff ${ }_{2}$ level would have been normal.

The pressure prescribed for the home CPAP setting was significantly less than Peff $_{1}$ but the total time allowed to reach $\mathrm{Peff}_{2}$ was 5.3 (1.5) hours. It can be anticipated that this titration procedure could not be achieved easily in a split night titration sleep study. According to the literature, the mean time allowed for the CPAP titration procedure during a split night procedure is 2.2 (1.0) hours. ${ }^{14}$ Furthermore, this procedure provides an inadequate measurement of the CPAP setting in $49 \%$ of patients, mainly because of the short duration of the CPAP titration. ${ }^{14}$ It is therefore reasonable to assume that this procedure could not be completed during a split night titration sleep study. 
The observed difference in Peff $_{1}$ and Peff 2 could be accounted for by a hysteresis phenomenon. Theory about hysteresis of the upper airway suggests that the intrinsic structure of the soft tissues which support the airway would lead to time dependent or pressure history dependent conformational changes, depending on the pressure levels applied. These characteristics of the upper airway could account for the significant decrease in effective pressure observed at the initial effective pressure level. The positive correlation between Peff $_{1}$ and Peff $_{1}-$ Pmin suggests that the amount of hysteresis of the upper airway is partly accounted for by the mechanical properties of the upper airway such as collapsibility. It is interesting to note that the difference in Peff was only observed in patients not previously treated with CPAP. Since collapsibility of the upper airways is known to decrease during treatment with $\mathrm{CPAP},{ }^{26}$ possibly as the result of a decrease in mucosal water content,${ }^{27}$ it can be speculated that such treatment could also modify hysteresis of the upper airway or any other upper airway characteristics that accounted for our findings.

Home oximetry recording is part of the standard screening procedure of patients referred to our Sleep Centre with a suspicion of SAHS. This has been shown to have a high diagnostic value ${ }^{20}$ and to be highly cost effective. ${ }^{28}$ The effective pressure level was significantly higher in patients diagnosed by home oximetry than in those diagnosed by polysomnography. Differences in BMI and neck circumference could be responsible for this difference in Peff between the two groups since these two factors are important determinants of Peff. ${ }^{29}$

It is interesting to place the present results in the context of automatic CPAP titration procedures. Some studies have shown that automatic titration may adequately determine the effective pressure level during hospital recordings. ${ }^{18}{ }^{24}$ Depending on the auto-CPAP titration algorithm, automatic titration procedures may occasionally find a higher effective pressure level than the manual titration procedure, ${ }^{19}{ }^{30}$ but this could be attributed to differences in the parameters analysed to modify the pressure setting. However, when using similar parameters, the manual pressure setting determined with a titration protocol similar to the one used in the present study is identical to that obtained from positive pressure trend provided by an auto CPAP machine. ${ }^{19}$ Since the auto CPAP pressure response is supposed to be automatically and continuously adapted to varying pressure requirements, the auto titration procedure may provide a more physiological estimate of Peff. Therefore, if the CPAP titration is performed manually, the titration procedure should include a decrement pressure trial in order to give a more realistic estimate of positive pressure needs.

It can be asked to what extent overtitrating CPAP could affect compliance with treatment. We are not aware of any study comparing CPAP compliance in patients using an exces- sively high CPAP level with those receiving an adequate CPAP level. On the other hand, we have previously shown that compliance with CPAP is better when these patients use an automatic CPAP which allows a decrease in the mean CPAP level. ${ }^{15}$ Furthermore, it is our clinical experience that, when possible (such as following weight loss), a decrease in the prescribed CPAP level improves pressure tolerance and mask comfort and decreases the risk of mask leaks. We therefore strongly believe that a fine adjustment in the CPAP level to the individual patient's need can only improve adherence to treatment.

In conclusion, our results show that downward titration during conventional determination of the effective pressure allows a significant decrease in the recommended pressure setting. The hysteresis phenomenon could be responsible for this difference. These findings should be taken into account during determination of the effective pressure for patients undergoing CPAP titration.

Supported by the Medical Research Council of Canada, Grant MT 13768 .

1 Young T, Palta M, Dempsey J, et al. The occurrence of sleep-disordered breathing among middle-aged adults. $N$ Engl f Med 1993;328:1230-5.

2 Partinen M, Guilleminault C. Daytime sleepiness and vascular morbidity at seven-year follow-up in obstructive sleep apnea patients. Chest 1990;97:27-32.

3 Kales A, Caldwell AB, Cadieux RJ, et al. Severe obstructive sleep apnea. II : Associated psychopathology and psychosleep apnea. II : Associated psychopathology and psy

social consequences. F Chronic Dis 1985;38:427-34.
4 Millman RP, Fogel BS, NcNamara ME, et al. Depression as a manifestation of obstructive sleep apnea: reversal with nasal continous positive airway pressure. f Clin Psychiatry 1989;50:348-51.

$5 \mathrm{He}$ J, Kryger MH, Zorick FJ, et al. Mortality and apnea index in obstuctive sleep apnea. Experience in 385 male patients. Chest 1988;94:9-14

6 Smith PL, Gold AR, Meyers DA, et al. Weight loss in mildly to moderately obese patients with obstructive sleep apnea. Ann Intern Med 1985;103:850-5.

7 Fujita S, Conway WA, Zorick FJ, et al. Evaluation of the effectiveness of uvulopalatopharyngoplasty. Laryngoscope 1985;95:70-4.

8 O'Sullivan RA, Hillman DR, Mateljan R, et al. Mandibular advancement splint: an appliance to treat snoring and obstructive sleep apnea. Am $f$ Respir Crit Care Med 1995;151:194-8.

9 Sullivan CE, Issa FG, Berthon-Jones M, et al. Reversal of obstructive sleep apnea by continuous positive airway pressure applied through the nares. Lancet 1981;i:862-5.

10 Waldhorn RE, Herrick TW, Nguyen MC, et al. Long-term compliance with nasal continuous positive airway pressure therapy of obstructive sleep apnea. Chest 1990;97:33-8

11 Hoffstein V, Viner S, Mateika S, et al. Treatment of obstructive sleep apnea with nasal continuous positive airway pressure. Patient compliance, perception of benefits, and side effects. Am Rev Respir Dis 1992;145:841-5.

12 Kribbs NB, Pack AI, Kline LR, et al. Effects of one night without nasal CPAP treatment on sleep and sleepiness in patients with obstructive sleep apnea. Am Rev Respir Dis 1993;147:1162-8.

13 Yamashiro Y, Kryger MH. CPAP titration for sleep apnea using a split-night protocol. Chest 1995;107:62-6.

14 Sanders MH, Kern NB, Costantino JP, et al. Adequacy of prescribing positive airway pressure therapy by mask for prescribing positive airway pressure therapy by mask for sleep apnea on the basis of a
Respir Dis 1993;147:1169-74.

15 Meurice J-C, Marc I, Sériès F. Efficacy of auto-CPAP in the treatment of obstructive sleep apnea/hypopnea syndrome. Am 7 Respir Crit Care Med 1996;153:794-8.

16 Condos R, Norman RG, Krishnasamy I, et al. Flow limitation as a noninvasive assessment of residual upper-airway resistance during continuous positive airway pressure therapy of obstructive sleep apnea. Am $\mathcal{F}$ Respir Crit Care Med 1994;150:475-80.

17 Montserrat JM, Alarcon A, Lloberes P, et al. Adequacy of prescribing nasal continuous positive airway pressure therapy for the sleep apnea/hypopnea syndrome on the basis of night time respiratory recording variables. Thorax 1995;50:969-71.

18 Ficker JH, Wiest GH, Lehnert G, et al. Evaluation of an auto-CPAP device for treatment of obstructive sleep apnea. Thorax 1998;53:643-8.

19 Lloberes P, Ballester E, Montserrat JM, et al. Comparison of manual and automatic CPAP titration in patients with 
sleep apnea/hypopnea syndrome. Am 7 Respir Crit Care Med 1996;154:1755-8.

20 Ryan PJ, Hilton MF, Boldy DAR, et al. Validation of British Thoracic Society guidelines for the diagnosis of sleep apnoea/hypopnoea syndrome. Thorax 1995;50:972-5.

21 Sériès F, Marc I, Cormier Y, et al. Utility of nocturnal home oximetry for case finding in patients with suspected sleep apnea-hypopnea syndrome. Ann Intern Med 1993;119: 449-53.

22 American Sleep Disorders Association. EEG arousals: scoring rules and examples. Sleep 1992;15:174-83.

23 Loube DI, Gay PC, Strohl KP, et al. Indications for positive pressure treatment of adult obstructive sleep apnea patients. Chest 1999;115:863-6.

24 Teschler H, Farhat AA, Exner V, et al. AutoSet nasal CPAP titration: constancy of pressure, compliance and effectiveness at 8 month follow-up. Eur Respir ₹ 1997;10:2073-8.

25 Stradling JR, Barbour C, Pitson DJ, et al. Automatic nasal continuous positive airway pressure titration in the continuous positive airway pressure titration in
laboratory: patient outcomes. Thorax 1997;52:72-5.
26 Schwartz A, Gold AR, Schubert N, et al. Effect of weight loss on upper airway collapsibility in obstructive sleep apnea. Am Rev Respir Dis 1991;144:494-8.

27 Ryan CF, Lowe AA, Li D, et al. Magnetic resonnance imagng of upper airway in obstructive sleep apnea before and after chronic nasal continuous positive airway pressure therapy. Am Rev Respir Dis 1991;144:939-44.

28 Chiner E, Sines-Costa J, Aiero LM, et al. Nocturnal oximetry for the diagnosis of the sleep apnea hypopnoea try for the diagnosis of the sleep apnea hypopnoea syndrome: a method to reduce the
graphies. Thorax 1999;54:968-71.

29 Sériès F, Marc I. Efficacy of automatic continuous positive airway pressure therapy that uses an estimated required pressure in the treatment of the obstructive sleep apnea syndrome. Ann Intern Med 1997;127:588-95.

30 Teschler $\mathrm{H}$, Berthon-Jones $\mathrm{M}$, Thompson $\mathrm{AB}$, et al. Automated continuous positive airway pressure titration for obstructive sleep apnea syndrome. Am $\mathcal{A}$ Respir Crit Care Med 1996;154:734-40. 